\title{
Factores asociados al desempeño académico según nivel de formación pregrado y género de los estudiantes de educación superior Colombia
}

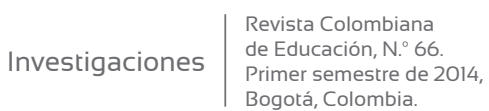

Bogotá, Colombia.
//Factors associated to academic

performance according to level of

education and gender among colombian

higher education students

//Fatores associados ao desempenho

académico segundo o nível de formação

de graduação e o sexo dos estudantes

de educação superior Colômbia

\section{Carla Ramírez*}

Economista con master en Educación Superior y candidata a doctor en Ciencias Sociales de la Universidad de Kassel Alemania. Correo electrónico: ramirezcarla@gmx.de

\section{Resumen}

El presente artículo tiene como objetivo analizar los factores socio-económicos y educativos asociados con el desempeño académico según nivel de formación y género de los estudiantes que presentaron la prueba Saber Pro 2009. Para lo anterior, se utilizaron bases de datos suministradas por el ICFES, de las cuales se conformó una base de datos de trabajo con 4.031 observaciones. Inicialmente, se realiza un análisis de tipo descriptivo utilizando distribuciones de frecuencia y se continúa con un análisis inferencial, usando pruebas de hipótesis, para verificar la independencia entre variables. Posteriormente, se utiliza un análisis de regresión lineal múltiple para determinar los factores asociados al desempeño académico, según género y nivel de formación, de los estudiantes evaluados con la prueba Saber Pro 2009. Se concluye que el desempeño académico previo medido en los puntajes obtenidos en las pruebas Saber 11 está asociado fuerte y positivamente con el de desempeño de los estudiantes sin importar su género o nivel de formación. Por su parte, las variables socio-económicas y educativas estudiadas resultaron, en comparación con el desempeño previo, estar débilmente asociadas con el desempeño en la educación superior.

\section{Abstract}

The present paper analyzes the socioeconomic and educational factors associated to the academic performance of students who took the Saber Pro exam in 2009, according to gender and level of education. With this purpose in mind, certain data bases from ICFES were used and a data set with 4.031 observations was created. First, a descriptive analysis using frequency distributions was carried out. Next, the independence of variables was tested using the Pearson's chi-squared test. Then, a multiple linear regression analysis was conducted to determine the factors associated to the student's academic performance according to gender and level of education. It was concluded that previous academic performance, measured by Saber 11 scores, is strongly and positively associated with the performance of higher education students, regardless their gender and level of education. On the other hand, socioeconomic and educational variables are weakly associated with the students' performance in higher education as compared to their previous performance in high school education.

\section{Palabras Clave}

Educación superior, educación técnica, educación tecnológica, Saber Pro, Colombia, desempeño académico.

\section{Keywords}

Higher education, technical education, technological education, Saber Pro, Colombia, academic performance, ICFES.

\section{Palavras chave}

Educação superior, educação técnica, educação tecnológica, Saber Pro, Colômbia, desempenho acadêmico. 


\section{Resumo}

Este estudo teve como objetivo analisar os fatores socioeconômicos e educacionais associados ao desempenho acadêmico segundo o nível de formação e o sexo dos estudantes que apresentaram a prova Saber Pro 2009. Utilizaram-se bancos de dados fornecidos pelo ICFES, a partir dos que se gerou um banco de dados de trabalho com 4031 observações. Inicialmente, foi feita uma análise descritiva usando distribuições de frequência, e continuou-se com análises inferenciais usando teste de hipótese para verificar a independência das variáveis. Foi feita uma análise de regressão múltipla para determinar os fatores associados ao desempenho acadêmico segundo o sexo e o nível de formação dos estudantes avaliados com a prova Saber Pro 2009. Concluiu-se que o desempenho acadêmico prévio avaliado com os escores obtidos nas provas Saber 11 têm uma associação positiva forte com o desempenho dos estudantes, não importando o sexo ou o nível de formação. Por outro lado, as variáveis socioeconômicas e educacionais estudadas mostraram estar debilmente associadas com o desempenho na educação superior.

Durante los años sesenta y setenta del siglo pasado, el estudio acerca del rendimiento académico sufrió grandes cambios en términos de metodología, enfoque y alcance. Para este momento, a los trabajos de psicólogos y educadores se le unen los estudios realizados por sociólogos y economistas, quienes introducen nuevas metodologías de investigación, como el uso de diferentes métodos de recolección de datos y técnicas de análisis multivariante (Schiefelbein y Simmons, 1981).

Con el pasar de los años, los estudios sobre el tema se han hecho más frecuentes no solo en el ámbito de educación básica, sino que se ha extendido a la educación superior. En Latinoamérica, se pueden mencionar los trabajos de Garbanzo (2007), quien analiza los factores asociados al rendimiento académico en estudiantes universitarios y su vinculación con la calidad de la educación pública, para lo cual se apoya en estudios realizados en España, Colombia, Costa Rica, Cuba y México; por su parte, Armenta, Pacheco y Pineda (2008) determinan la causa del bajo rendimiento académico de los estudiantes universitarios de la Facultad de Ciencias Humanas de la Universidad Autónoma de Baja California (UABC) y Ferreyra (2007) analiza la influencia de las características personales, el entorno familiar, el desempeño en la escuela secundaria y la etapa inicial de la vida universitaria sobre el rendimiento de la cohorte 2002 de la Facultad de Ciencias Económicas de la Universidad Nacional de La Plata de Argentina.

Igualmente, es importante resaltar que la literatura sobre rendimiento académico es muy variada, así como lo son los factores que potencialmente pueden influenciar el desempeño de los 
estudiantes (Hernández, Márquez, y Palomar, 2006). Respecto a esto, vale la pena mencionar que entre los temas que han sido ampliamente estudiados, a nivel internacional, está la manera en que las diferencias de género influyen en el desempeño académico de los estudiante (Nowel y Hedges, 1998) y acerca de la asociación existente entre el desempeño académico en la educación secundaria y el desempeño en la educación superior; algunos ejemplos son Conger y Long (2010) y Türüt-Asık y Meltem (2007).

En Colombia, algunos estudios sobre el tema son los siguientes: Villalba y Salcedo (2008), quienes analizan cómo el rendimiento en la educación media está asociado al rendimiento académico en la universidad; Vélez y Roa (2005) y Garzón, Rojas, Del Riesgo y Salamanca (2010), quienes estudian a los estudiantes de la Facultad de Medicina de la Universidad del Rosario. El primer estudio estuvo dirigido a los estudiantes de medicina de primer semestre y su objetivo era determinar los factores que estaban asociados a su desempeño, mientras que el segundo trabajo analizó los factores que pueden influir en el rendimiento académico de estudiantes de Bioquímica que ingresan en el programa de Medicina de esta universidad.

En lo que respecta a estudios que aborden el tema educación superior técnica profesional y tecnológica son limitados en comparación con investigaciones sobre la edu- cación superior universitaria, por lo que estudios que se enfoquen en el rendimiento de los estudiantes de estos programas académicos, técnicos profesionales y tecnológicos son aún más escasos. De hecho, Gómez (2003), Martínez, Asmar, Silva y Toro (2005) y Ramírez (2008) han señalado que, a pesar de que el sector no universitario, entiéndase carreras técnicas profesionales y tecnológicas, ha pasado a tener un rol significativo en las políticas nacionales, los estudios sobre el sector son escasos. Y en los tiempos actuales, donde precisamente se promueve el aumento de la cobertura del sistema terciario mediante este tipo programas, es vital tener mayor información sobre el desempeño de sus estudiantes.

En este contexto, la presente investigación pretende analizar los factores que afectan el rendimiento académico en la educación terciaria, según género y nivel de formación pregrado, es decir, nivel técnico profesional, tecnológico y profesional. Ambos aspectos no solo son relevantes debido a la relativa escases de estudios sobre el tema, ya señalada por diferentes autores, sino que también son pertinentes dada las actuales políticas nacionales de educación superior, en la cuales se promueve la expansión del sistema de educación terciaria mediante la promoción de los programas técnicos profesionales y tecnológicos.

Resumiendo, la investigación tiene como objetivo general analizar 
los factores socio-económicos y educativos que están asociados con el buen desempeño de los estudiantes que presentaron el examen de estado de la calidad de la educación superior, Saber Pro', en 2009, según género y nivel de formación pregrado.

\section{Metodología}

Teniendo en cuenta que uno de los principales objetivos de esta investigación es analizar por nivel de formación, la selección de la muestra se hizo teniendo en cuenta que estuvieran presentes estudiantes de los tres niveles de formación de la educación superior, técnico profesional, tecnólogo y profesional.

Se utilizaron las bases de datos del Icfes (Instituto Colombiano para la Evaluación de la Educación), una entidad especializada en ofrecer servicios de evaluación de la educación en todos sus niveles, y en particular apoyar al Ministerio de Educación Nacional en la realización de los exámenes de estado y en adelantar investigaciones sobre calidad educativa (Icfes, 2014b).

Después de analizar estas bases de datos, se encontró que, con las características propuestas en esta investigación, solo correspondían los estudiantes que presentaron las pruebas específicas Saber Pro en administración, ingeniería de sistemas, ingeniería electrónica, tecnología en administración y afines, tecnología en sistemas y afines, tecnología en electrónica y afines, técnico profesional en administración y afines, técnico profesional en sistemas y afines, técnico profesional en electrónica y afines en 2009. Así las cosas, la población seleccionada para esta investigación corresponde a los estudiantes que presentaron las pruebas antes mencionadas y que además realizaron el examen Saber $11^{2}$ en 2005 y 2006.

1 El Examen de Estado de Calidad de la Educación Superior, Saber Pro, es un instrumento estandarizado para la evaluación externa de la calidad de la educación superior que evalúa las competencias genéricas, que son las necesarias para el adecuado desempeño profesional, independientemente del programa cursado y las competencias específicas que contemplan elementos disciplinares fundamentales de la formación superior que son comunes a grupos de programas en una o más áreas del conocimiento. Este examen es de carácter obligatorio y está dirigido a los estudiantes de pregrado de programas técnicos profesionales, tecnológicos y profesionales universitarios que hayan aprobado por lo menos el $75 \%$ de los créditos académicos del programa que cursan (Icfes, 2014a)

2 Saber $11^{\circ}$ es el examen de Estado de la educación media colombiana, cuyo fin es comprobar el grado de desarrollo de las competencias de los estudiantes que están por terminar undécimo grado. Igualmente lo presentarán aquellas personas que hayan obtenido el título de bachiller o hayan superado el examen de validación del bachillerato. Los resultados oficiales son un requisito para el ingreso a la educación superior (Icfes, 2014c). 
La anterior restricción, es decir, estudiantes que presentaron la prueba Saber Pro y a su vez la prueba Saber 11, se hizo en razón de incluir variables relevantes que ofrecieran información acerca de los antecedentes educativos de los estudiantes. La selección de 2005 y 2006 fue resultado de la información disponible en las bases de datos del Icfes que además fuera comparativa y solamente lo años mencionados cumplían con esas dos condiciones.

Después de cruzar la información de las diferentes bases de datos suministradas por el Icfes, se obtiene finalmente una población con 4.031 observaciones.

Con base en los datos antes descritos, se realiza un análisis de tipo descriptivo utilizando distribuciones de frecuencia. Posteriormente, se realiza un análisis de regresión lineal múltiple para determinar los factores asociados al desempeño académico, según género y nivel de formación, de los estudiantes evaluados con la prueba Saber Pro 2009. Para lo anterior, se realizan regresiones independientes por nivel de formación pregrado, es decir, regresiones para el grupo de técnicos profesionales, tecnólogos y profesionales; igualmente, se hacen estimaciones tanto para hombres como para mujeres por separado.

\section{Principales resultados}

Es importante resaltar que los resultados obtenidos son aplicables a la muestra estudiada y de ninguna manera pretenden generalizar a la población colombiana.

Los resultados presentados a continuación se obtienen del análisis de tablas de frecuencia, en las cuales se utilizaron como variables de referencia el género y el nivel de formación pregrado de los estudiantes.

\section{Características de}

\section{los encuestados}

Después de realizar los cruces de información correspondientes, se obtuvo una base de datos final con 4.031 individuos, de los cuales 2.145 son mujeres $(53.2 \%)$, mientras que 1.886 son hombres (46.8\%); $95 \%$ de ellos son solteros y la edad promedio al momento de realizar la prueba Saber Pro 2009 es 21 años.

La mayor presencia de mujeres se podría explicar entre otras por las siguientes razones: 1) la proporción de mujeres matriculadas en el sistema de educación superior es relativamente mayor a la de hombres. En 2009, el $52 \%$ de estudiantes matriculados en este nivel eran mujeres y el $48 \%$ eran hombres (UCC, 2010) y 2) las áreas de conocimiento estudiadas, entiéndase administración y afines, ingeniería de sistemas e ingeniería electrónica, pudo haber influido en la composición de la población. Particularmente, la primera de ellas, administración y afines, se caracteriza no solo por ser una de las áreas más demandadas dentro del sistema, sino también por contar con una alta participación de mujeres. 
Según el tipo de prueba presentada, se puede concluir que la participación de los estudiantes que realizaron la prueba Saber Pro en 2009 y a su vez realizaron la prueba Saber 11 en 20052006, de acuerdo con el nivel de formación, es la siguiente: 15\% son estudiantes de programas técnicos profesionales, 63\% son de programas tecnológicos y $22 \%$ son de programas profesionales (tabla1).

Tabla 1. Número total de estudiantes de las áreas escogidas que presentaron la prueba Saber Pro 2009 y a su vez la prueba Saber 11 en 2005 2006 según nivel de formación.

\begin{tabular}{|c|c|c|}
\hline Nivel de formación & Frecuencia & $(\%)$ \\
\hline Profesional & 890 & 22 \\
\hline Tecnológica & 2.557 & 63 \\
\hline Técnica profesional & 584 & 15 \\
\hline Total & 4.031 & 100 \\
\hline
\end{tabular}

Inicialmente, se pensaría que el resultado obtenido, según nivel de formación, difiere de la realidad, especialmente si se tiene en cuenta que en 2009, del total de matriculados en las áreas de administración, ingeniería de sistemas e ingeniería electrónica, el 64\% estaba matriculado en programas profesionales, $28 \%$ en programas tecnológicos y el $8 \%$ en programas técnicos profesionales; sin embargo, dada la disponibilidad de información y las restricciones hechas, la población bajo estudio cuenta con una mayor representación de estudiantes de programas tecnológicos. Esta situación se explica por el hecho de que las pruebas Saber Pro se presentan durante los semestres finales de los programas académicos y considerando que la duración de los programas de cada uno de los niveles de formación es diferente (dos, tres, cuatro y cinco años), no es de extrañar la mayor representación de estudiantes de programas tecnológicos, pues ellos serían los estudiantes que de acuerdo con el periodo entre la prueba Saber 11 (2005 y 2006) y la prueba Saber Pro (2009) estarían en los últimos semestres de sus programas académicos, cuya duración está entre tres y cuatro años.

Además, se observa una clara diferencia en la participación de estudiantes según estrato socio-económico, predominando aquellos de estratos bajos. De hecho, la composición de la población obtenida según estrato es similar a la distribución socio-económica del país, donde, según la Encuesta de Calidad de Vida de 
2003, el 89\% de la población colombiana pertenece a los estratos uno, dos y tres (citado en Conpes 3386, 2005). Mientras que en la población aquí estudiada el $84 \%$ de los estudiantes pertenece a estos estratos, el porcentaje restante, $15 \%$, se distribuye en los tres estratos más altos.

Asimismo, se puede decir que el nivel de educación de las madres es comparativamente menor al de los padres. Por un lado, $69 \%$ de los padres de los estudiantes no ha realizado estudios de nivel superior, es decir, no tiene ningún tipo de estudios o tiene algún tipo de estudios primarios o secundarios; mientras tanto, el porcentaje para las madres es de $74 \%$. A pesar de este hecho, se puede decir que dentro del grupo de padres sin estudios terciarios, un porcentaje mayor de madres alcanzó algún tipo de estudios secundarios, en comparación con los padres (tabla2).
En lo que corresponde a las características educativas de la muestra estudiada, se pueden destacar las siguientes:

1. Dos tercios de los estudiantes terminaron sus estudios secundarios en planteles clasificados por el Icfes como altos y solo un $7 \%$ lo hicieron en planteles clasificados como bajos. Este resultado es para reflexionar. ¿Significa esto que el sistema terciario de educación es de difícil acceso para los estudiantes graduados de instituciones clasificadas como de categoría baja? ¿O que los estudiantes de los colegios de esa categoría sí entran a la educación superior, pero desertan y por tanto su poca participación en esta muestra?

Tabla 2. Nivel de educación alcanzado por el padre y la madre de los estudiantes que presentaron la prueba Saber Pro 2009 y a su vez la prueba Saber 11 en 2005 y 2006.

\begin{tabular}{|l|c|c|c|c|}
\hline & \multicolumn{2}{|c|}{ Padre } & \multicolumn{2}{c|}{ Madre } \\
\hline & Frecuencia & $\%$ & Frecuencia & $\%$ \\
\hline Algún tipo de estudios primarios & 1.034 & 25.7 & 929 & 23.0 \\
\hline Algún tipo de estudios de bachillerato & 1.612 & 40.0 & 1.870 & 46.4 \\
\hline Algún tipo de educación técnica/tecnológica & 505 & 12.5 & 525 & 13.0 \\
\hline Algún tipo de educación profesional & 584 & 14.5 & 493 & 12.2 \\
\hline Posgrado & 166 & 4.1 & 147 & 3.6 \\
\hline Ninguno & 130 & 3.2 & 39 & .7 \\
\hline No sabe & & & 28 & 1.0 \\
\hline Total & 4.031 & 100.0 & 4.031 & 100.0 \\
\hline
\end{tabular}


2. Más de la mitad de los estudiantes, $60 \%$, terminaron sus estudios de bachillerato en planteles públicos.

3. $55 \%$ presentaron la prueba de administración y afines, $31 \%$ de ingeniería de sistemas y afines y $14 \%$ de ingeniería electrónica y afines.

4. $64 \%$ de los estudiantes se encontraban matriculados en una IES de origen privado, mientras el $36 \%$ en una IES de origen público. El anterior resultado no dista mucho de las estadísticas nacionales de matriculados en educación superior por área de conocimiento. De hecho, en $2009,53 \%$ de los estudiantes matriculados en las áreas aquí analizadas se encontraban matriculados en una institución de educación superior privada, mientras el $47 \%$ se encontraba matriculado en una institución pública. Este resultado es especialmente interesante porque difiere de las estadísticas generales de la educación superior; en los últimos diez años, la proporción de matriculados en el sector público ha ido aumentando, y específicamente desde 2006 esta ha sido mayor que la de estudiantes matriculados en el sector privado (SNIES, 2012). Entonces, el resultado obtenido podría significar que especialmente en estas áreas del conocimiento la oferta de programas es mayor en el sector privado que en el público, pero también podría indicar que la demanda, particularmente en estas áreas, prefiere la educación privada a la pública;

5. Solo el $10 \%$ de los estudiantes aquí estudiados cursaba estudios en una IES acreditada, mientras el 17\% de ellos estudiaba un programa acreditado. Es importante recordar que la acreditación en Colombia es de carácter voluntario, lo que puede explicar la relativa baja presencia de estudiantes en programas e instituciones acreditadas.

\section{Factores asociados con el desempeño académico}

Este apartado se divide en tres subsecciones, en la primera se explica el modelo de análisis utilizado y la segunda y tercera parte presentan los resultados obtenidos según género y nivel de formación. 


\section{Modelo de análisis}

Con el propósito de identificar las variables socio-económicas y educativas (variables independientes) asociadas con el desempeño académico (variable dependiente) de los estudiantes de administración, ingeniería de sistemas, ingeniería electrónica, tecnología en administración y afines, tecnología en sistemas y afines, tecnología en electrónica y afines, técnico profesional en administración y afines, técnico profesional en sistemas y afines, técnico profesional en electrónica que presentaron el examen Saber Pro en 2009 se utiliza el análisis de regresión múltiple.

Se realizan regresiones independientes por nivel de formación, es decir, regresiones para el grupo de técnicos profesionales, tecnólogos y profesionales; igualmente, se hacen estimaciones tanto para hombres como para mujeres por separado. La entrada de datos se hace por el método de bloque y se excluyen aquellas variables con menos de diez casos. Inicialmente, se introducirán aquellas variables con mayor grado de correlación, por ejemplo, puntaje prueba Saber 11, al igual que las variables género y tipo de programa; seguidamente, se introducirán las variables relacionadas con las características socio-económicas, características de educación superior y características de la educación secundaria, en ese orden.

Para efectos de mejor ilustración, el valor de beta se describió de 0.10 o menor como débil, mayor que 0.10 pero menor que 0.20 como moderado, y 0.20 o mayor como fuerte (Duarte y Galaz, 2006).

Asimismo, a partir de las variables de tipo categórico se crearon variables dummy y se utiliza como variable de referencia aquella con mayor frecuencia.

Vale la pena mencionar que el grupo de variables usado para describir las características de la educación superior de los técnicos profesionales difiere de las variables usadas en el grupo de tecnólogos y profesionales. En vez de seis variables, se utilizan tres para describir las características de la educación superior, es decir, se omiten las variables institución acreditada (iesac) y programa acreditado (proac) por falta de datos; además, al no haber un número suficiente de casos para la variable dummy universidad (uni), se decide agrupar esta variable junto con institución universitaria, quedando así solo dos variables dummy que representan el tipo de institución de educación superior. Igualmente, la variable referencia para esta variable pasa a ser instituto técnico profesional (ITP) y no universidad, como ocurre para el grupo de tecnólogos y profesionales.

\section{Según género}

En el análisis por género, se observa que el desempeño académico previo de los estudiantes está asociado positivamente con su desempeño durante los estudios superiores 
(tabla 3). Para los hombres, en términos generales, los puntajes obtenidos en las diferentes áreas del examen Saber 11 están asociados moderadamente con desempeño en la educación superior; cinco de los siete indicadores tienen betas entre 0.1 y 0.2. Por otro lado, para las mujeres, la asociación de acuerdo con el área del examen es variada, por un lado, lenguaje y biología están asociadas fuertemente, betas $=.220$ y .208 , respectivamente; mientras que química, física, matemáticas e idiomas se encuentran asociadas débilmente, betas menores que 0.1 .

Igualmente, se encuentra que el tipo de programas estudiado está asociado con su desempeño. Cursar un programa profesional en vez de un programa tecnológico o técnico profesional está asociado fuertemente y positivamente con el desempeño de ambos tipos de estudiantes, hombres y mujeres.

Tabla 3. Resumen de resultado de análisis de regresión múltiple por género.

\begin{tabular}{|c|c|c|c|c|c|c|}
\hline \multirow{2}{*}{ VIs } & \multicolumn{3}{|c|}{ Hombres } & \multicolumn{3}{|c|}{ Mujeres } \\
\hline & Beta & T-Value & Sig & Beta & T-Value & Sig \\
\hline (Constant) & & 7.035 & .000 & & 11.689 & .000 \\
\hline TEMA_QUIMICA & .142 & 6.058 & .000 & .099 & 4.792 & .000 \\
\hline TEMA_FISICA & .097 & 4.497 & .000 & .062 & 3.388 & .001 \\
\hline TEMA_BIOLOGIA & .199 & 8.430 & .000 & .208 & 9.797 & .000 \\
\hline TEMA_FILOSOFIA & .090 & 4.590 & .000 & .123 & 6.483 & .000 \\
\hline TEMA_MATEMATICA & .120 & 5.498 & .000 & .091 & 4.890 & .000 \\
\hline TEMA_LENGUAJE & .173 & 7.924 & .000 & .220 & 10.726 & .000 \\
\hline TEMA3_IDIOMA_P & .129 & 5.408 & .000 & .094 & 3.989 & .000 \\
\hline progtecnovsproguni & .260 & 8.903 & .000 & .201 & 8.723 & .000 \\
\hline progtecvsproguni & .179 & 6.139 & .000 & .211 & 8.730 & .000 \\
\hline \multicolumn{7}{|l|}{ Socio-demográficas } \\
\hline estcivil1 & -.013 & -.721 & .471 & .039 & 2.267 & .023 \\
\hline Edad & -.061 & -3.127 & .002 & -.019 & -1.059 & .290 \\
\hline algunprimvsalgunbach $\mathrm{P}$ & .050 & 2.409 & .016 & .027 & 1.310 & .190 \\
\hline $\begin{array}{l}\text { alguntec/tecno vs } \\
\text { algunbach } \mathrm{P}\end{array}$ & -.009 & -.497 & .619 & -.024 & -1.287 & .198 \\
\hline algunprofvsalgunbach P & -.010 & -.491 & .623 & -.008 & -.393 & .695 \\
\hline posgradovsalgunbach $\mathrm{P}$ & -.012 & -.544 & .586 & .027 & 1.342 & .180 \\
\hline $\begin{array}{l}\text { ningunaeduvsalgunbach } \\
P\end{array}$ & -.009 & -.474 & .636 & -.021 & -1.173 & .241 \\
\hline algunprimvsalgunbach $\mathrm{M}$ & .015 & .748 & .454 & -.007 & -.369 & .712 \\
\hline
\end{tabular}




\begin{tabular}{|c|c|c|c|c|c|c|}
\hline $\begin{array}{l}\text { alguntec/tecno vs } \\
\text { algunbach } M\end{array}$ & .032 & 1.721 & .085 & -.004 & -.225 & .822 \\
\hline algunprofvsalgunbach M & -.009 & -.460 & .645 & -.007 & -.368 & .713 \\
\hline posgradovsalgunbach M & -.007 & -.366 & .714 & -.062 & -3.379 & .001 \\
\hline $\begin{array}{l}\text { ningunaeduvsalgunbach } \\
M\end{array}$ & .016 & .864 & .388 & -.007 & -.395 & .693 \\
\hline estmediovsestbajo & .016 & .827 & .409 & .037 & 1.923 & .055 \\
\hline estaltovsestbajo & -.026 & -1.161 & .246 & -.033 & -1.459 & .145 \\
\hline $\begin{array}{l}\text { ingfam-1sm vsingfam } \\
1-2 \mathrm{sm}\end{array}$ & -.051 & -2.862 & .004 & -.018 & -1.010 & .313 \\
\hline $\begin{array}{l}\text { ingfam2-3vs ingfam } \\
1-2 \mathrm{sm}\end{array}$ & -.027 & -1.380 & .168 & .040 & 2.109 & .035 \\
\hline $\begin{array}{l}\text { ingfam3-5 vsingfam } \\
1-2 \mathrm{sm}\end{array}$ & .015 & .760 & .447 & .071 & 3.643 & .000 \\
\hline $\begin{array}{l}\text { ingfam } 5 \text { o + vs ingfam } \\
1-2 \mathrm{sm}\end{array}$ & .073 & 3.110 & .002 & .107 & 4.785 & .000 \\
\hline \multicolumn{7}{|l|}{$\begin{array}{l}\text { Características edu- } \\
\text { cación superior }\end{array}$} \\
\hline orgn_ies & -.060 & -3.169 & .002 & -.037 & -1.937 & .053 \\
\hline lesac & -.016 & -.676 & .499 & -.005 & -.244 & .807 \\
\hline Progac & .034 & 1.646 & .100 & .014 & .702 & .483 \\
\hline InstUnivsUni & -.110 & -4.146 & .000 & -.044 & -1.714 & .087 \\
\hline Escuela/Inst. Tecno vs Uni & -.146 & -5.503 & .000 & -.135 & -5.548 & .000 \\
\hline InstTecProfvsUni & -.012 & -.490 & .624 & -.050 & -2.054 & .040 \\
\hline \multicolumn{7}{|l|}{$\begin{array}{l}\text { Características edu- } \\
\text { cación secundaria }\end{array}$} \\
\hline bachnormvsbachacad & .007 & .418 & .676 & .002 & .103 & .918 \\
\hline bachTecvsbachacad & .053 & 2.920 & .004 & -.011 & -.607 & .544 \\
\hline colenatu & .005 & .231 & .817 & .006 & .304 & .761 \\
\hline $\begin{array}{l}\text { colecatebajovscoleca- } \\
\text { tealto }\end{array}$ & .005 & .260 & .795 & .009 & .477 & .634 \\
\hline $\begin{array}{l}\text { colecatemediovscoleca- } \\
\text { tealto }\end{array}$ & .016 & .891 & .373 & -.019 & -1.072 & .284 \\
\hline R-Cuadrado Ajustado & 0.462 & & & 0.416 & & \\
\hline Observaciones & 1886 & & & 2145 & & \\
\hline
\end{tabular}

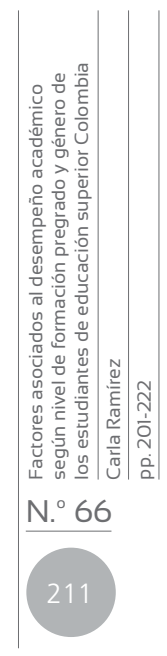

En cuanto a las variables socio-económicas, algunas diferencias entre hombres y mujeres se manifiestan. Para las mujeres, el estado civil está asociado positivamente, aunque de forma débil (beta $=.039$ ), con su desempeño; ser soltera está relacionado con un mejor desempeño; esto se puede deber a que las mujeres solteras tendrían más tiempo para dedicarle al estu- 
dio que el que le pueden dedicar las mujeres casadas, que deben alternar su rol como estudiante con el de esposa y madre.

Mientras tanto, para los hombres el estado civil resulta no estar asociado con su desempeño; aunque la edad sí. La edad está asociada débilmente (beta =-.061) en forma negativa con su desempeño académico.

El estrato no resulta ser una variable asociada con el desempeño académico de los hombres, pero sí para las mujeres. Pertenecer al estrato medio, en vez del estrato bajo, está asociado positivamente con su desempeño académico. Es decir, las mujeres en comparación con los hombres, necesitan ciertas condiciones económicas y sociales para tener un mejor desempeño.

En lo referente al ingreso familiar, para las mujeres, ingresos mayores o iguales a dos salarios mínimos y menores de cinco salarios mínimos están asociados positivamente y de forma débil (betas< 0.1) con su desempeño. Para los hombres, en cambio, solo los ingresos familiares o muy bajos, menores de un salario mínimo, o muy altos, mayores de cinco salarios mínimos, resultaron estar relacionados; el primero de ellos está asociado negativamente y el segundo de ellos, positivamente. Es de resaltar que tanto para hombres como para mujeres los ingresos superiores a cinco salarios mínimos están asociados positivamente.

En cuanto a las características de la educación superior, se encuentra que el tipo de institución influye en el desempeño tanto de hombres como mujeres; en general, estudiar en instituciones diferentes a la universidad afecta negativamente el desempeño de los estudiantes. Lo anterior se puede deber a las diferentes condiciones físicas y académicas que poseen los distintos tipos de instituciones.

En resumen, cuando se realizan las regresiones individuales por género, se encuentran algunas diferencias en las variables que resultaron ser estadísticamente significativas para uno y otro grupo. Con excepción del rendimiento en la educación secundaria, medido en los puntajes obtenidos en el examen Saber 11 en las áreas de química, física, matemáticas, biología, filosofía, lenguaje e idiomas; pocas variables resultaron estar asociadas con el desempeño en la educación superior de ambos tipos de estudiantes, hombres y mujeres: origen de la IES, estudiar en una escuela/institución tecnológica en vez de universidad, así como tener a ingresos familiares iguales o mayores a cinco salarios mínimos. 
Igualmente, vale la pena destacar que el tipo de asociación de las variables mencionadas con el desempeño en la educación superior es el mismo. El desempeño previo así como tener un ingreso familiar superior a cinco salarios mínimos están asociados positivamente con el desempeño en la educación superior; mientras que las otras dos variables, por ejemplo, origen de la IES y estudiar en una escuela/institución tecnológica en vez de una universidad está asociado negativamente.

\section{Por nivel de formación}

En los tres niveles de formación se confirma lo encontrado en otros estudios sobre el tema (Duarte y Galaz, 2006; Ferreyra, 2007; Villalba y Salcedo, 2008): el desempeño académico previo a la educación superior, en este caso medido en el puntaje de la prueba Saber 11 , está significativamente asociado con el desempeño durante los estudios superiores (tabla 4).

En cuanto al género, los resultados indican que esta variable está asociada débil y negativamente con el desempeño de los estudiantes de los niveles, tecnológico y profesional. Ser mujer tiene un efecto negativo en el puntaje de la prueba Saber Pro para los estudiantes de programas tecnológicos y profesionales.

Una posible razón para este resultado podría ser las áreas de conocimiento aquí estudiadas, ciencias administrativas e ingenierías. Investigaciones en Estados Unidos han señalado que existe una brecha entre el desempeño académico de hombres y mujeres en estas áreas, que no necesariamente tienen que ver con factores socio-demográficos o académicos de los estudiantes, pero sí con factores psicológicos y pedagógicos, los cuales podrían explicar la diferencia en el desempeño de hombres y mujeres en estas áreas del conocimiento (Blackman, 2011 y Felder et al. 1995).

Cuando se analizan las variables socio-económicas, algunas diferencias por nivel de formación se hacen presentes. La variable estrato socioeconómico está débil y negativamente asociada con desempeño de los estudiantes de programas profesionales. Pertenecer a un estrato alto en vez de uno bajo está relacionado negativamente con el desempeño académico de los estudiantes de programas profesionales. Una posible razón para este resultado puede ser las diferentes motivaciones que estudiantes de estos dos grupos puedan tener; por ejemplo, estudiantes de estratos socio-económicos bajos que cursan programas de tipo profesional pueden estar becados por su buen desempeño académico durante sus estudios de bachillerato para realizar estudios superiores. Por lo cual, además de tener buenos antecedentes académicos, el hecho de continuar recibiendo una beca para financiar sus estudios les motiva a mantener un alto desempeño. De hecho, $44 \%$ de los estudiantes de estrato bajo que cursan 


$$
\begin{aligned}
& \text { ஸे \& \& \& } \\
& \text { 눈 }
\end{aligned}
$$

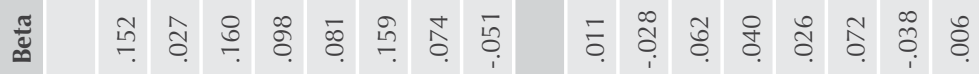

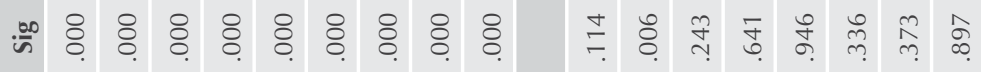

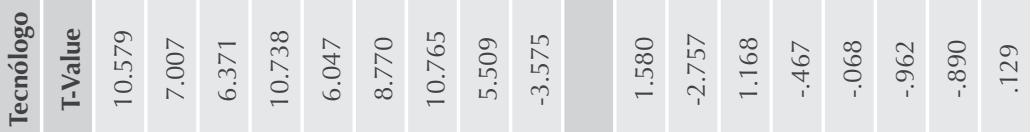

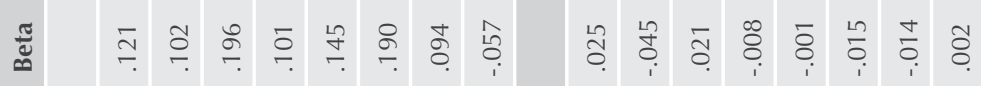

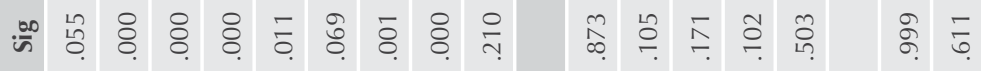

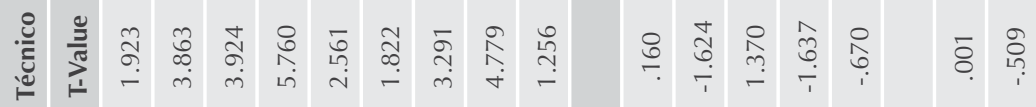

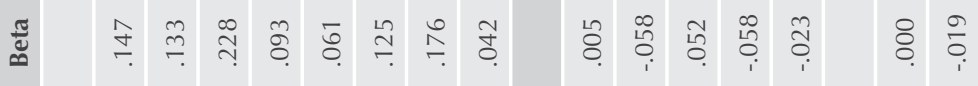




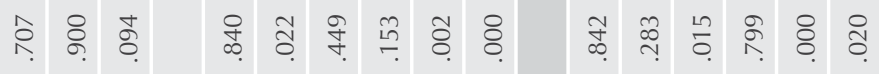

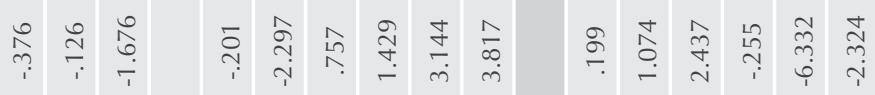

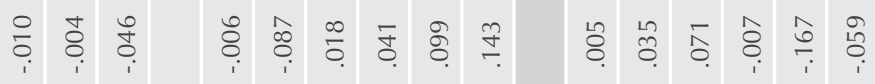

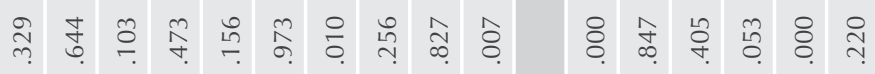

$$
\begin{aligned}
& \text { م. }
\end{aligned}
$$

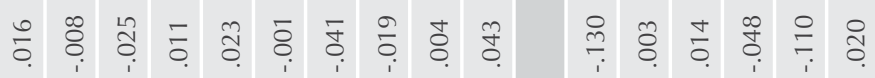

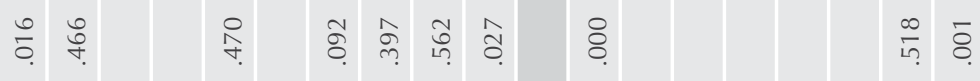

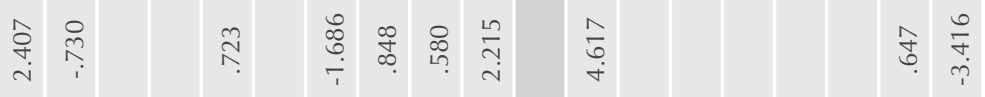

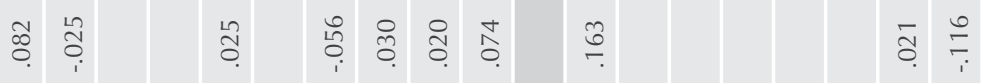

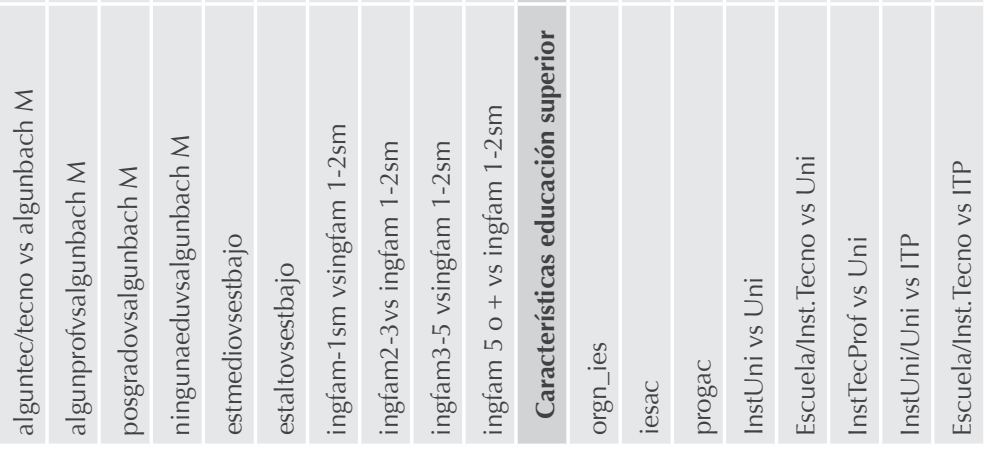

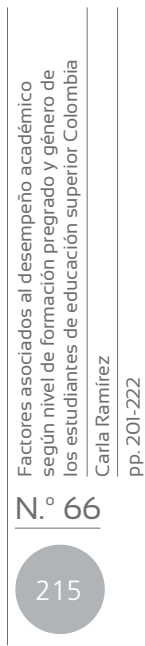




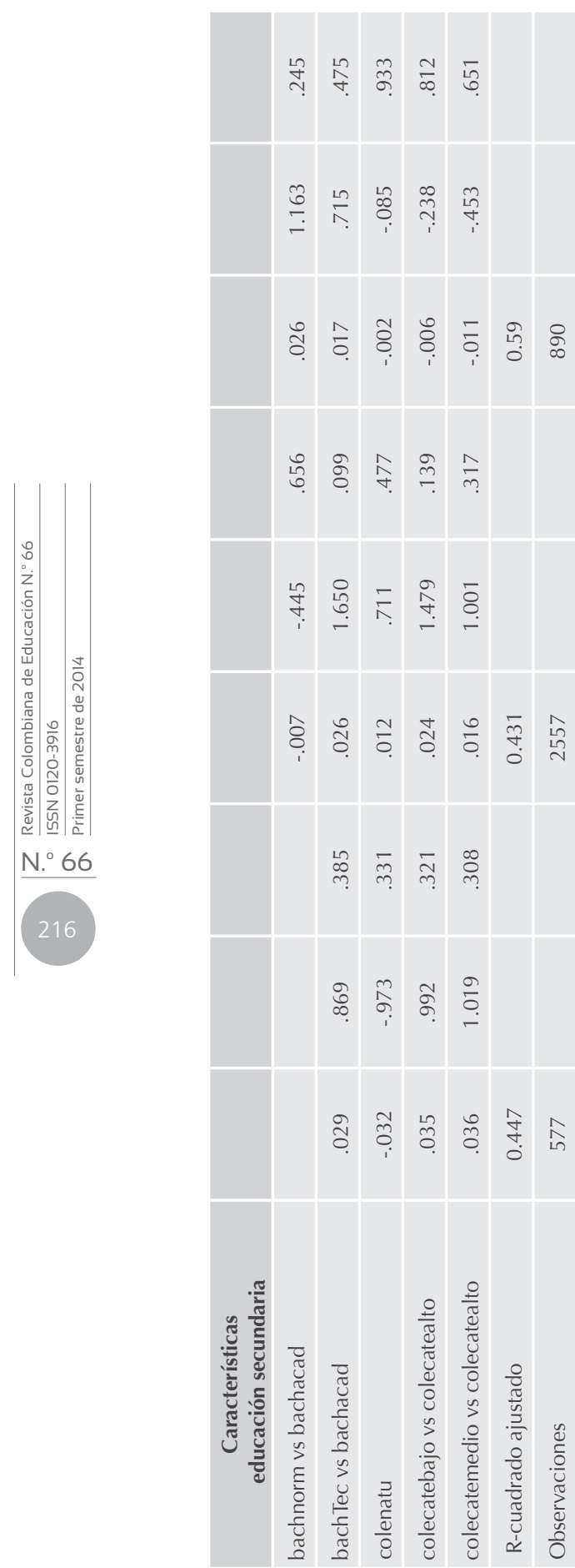

programas profesionales financia sus estudios por medio de una beca.

Además, como era esperado, altos ingresos familiares, iguales o mayores a cinco salarios mínimos, están asociados positivamente con el desempeño académico de los estudiantes de los tres niveles de formación. Para los estudiantes de programas técnicos profesionales (beta $=.074)$ y tecnológicos (beta=.043), la asociación es débil; mientras que para los estudiantes de programas profesionales, la asociación es moderada (beta=.143).

En cuanto a las variables relacionadas con las características de la educación superior, algunas diferencias de acuerdo con el nivel de formación se presentan. El origen de la IES, público o privado, está asociado moderadamente, aunque de manera diferente con el desempeño de los estudiantes que cursan programas técnicos profesionales y tecnológicos; para los primeros, la asociación es negativa, mientras que para los segundos positiva.

Igualmente, se encuentra una asociación moderada, pero diferente según el tipo de programas, entre el tipo de institución y el desempeño académico de los estudiantes. Estudiar en una universidad, en vez de una institución universitaria o un institución/escuela tecnológica está positivamente asociado con el desempeño de los estudiantes de los programas tecnológicos y profesionales. Por su parte, para los estudiantes de programas técnicos profe- 
sionales, el hecho de estudiar en una escuela/institución tecnológica, en vez de una institución técnica profesional (en adelante ITP), está asociado negativamente con su desempeño académico.

La anterior situación se puede deber a características propias de las instituciones. Por lo general, las ITP son instituciones especializadas donde principalmente se ofrecen programas técnicos profesionales, lo cual podría explicar el mejor desempeño de los estudiantes de estas instituciones comparado con el de las instituciones tecnológicas, las cuales tienen un alcance más amplio que las primeras. Asimismo, el mejor desempeño de estudiantes de programas tecnológicos y profesionales que estudian en universidades, en vez de instituciones tecnológicas, se puede deber a las características propias de las universidades, las cuales por lo general cuentan con instalaciones mejores dotadas y más cómodas, como también con mayores recursos académicos, que las que se puede encontrar en una institución/escuela tecnológica. De hecho, Ramírez (2012), en una encuesta realizada a graduados de educación superior de 2008 en áreas de administración y afines, salud e ingenierías del departamento del Atlántico encuentra que en la evaluación de las condiciones física y académica que hicieron los graduados sobre sus respectivas instituciones, las universidades obtuvieron los mejores puntajes.
Las variables relacionadas con la calidad de la educación superior, medidas en instituciones y programas acreditados, en términos generales no están asociadas con el desempeño académico de los estudiantes. Solo los programas profesionales acreditados resultaron estar asociados positivamente, de manera débil (beta=.071), con el desempeño de los estudiantes. Es decir, estudiar un programa profesional acreditado vale la pena si se compara con estudiar programas profesionales no acreditados; los primeros tendrían un mejor desempeño académico que los segundos.

Con excepción del desempeño académico del bachillerato, medido en el puntaje de la prueba Saber 11, las variables relacionadas con las características de la educación secundaria, entiéndase, categoría de la institución, naturaleza de la institución y título de bachillerato obtenido, resultaron no ser significativas para los estudiantes de ninguno de los tres niveles de educación superior. Este resultado es similar al encontrado por Garzón et al. (2010) al analizar los factores asociados con el rendimiento de los estudiantes de Bioquímica que ingresan al programa de Medicina de la Universidad del Rosario, donde halló que, mientras los resultados obtenidos en las pruebas Saber 11 estaban asociados positivamente y eran significativos estadísticamente con el rendimiento de los estudiantes, la categoría del colegio donde se realizaron los estudios secundarios no lo era. 
A manera de resumen, se puede decir que las variables relacionadas con el desempeño en la educación secundaria, puntajes Saber 11, están asociadas positivamente con el desempeño en la educación terciaria. Por el contrario, las variables relacionadas con la educación secundaria no resultaron estar relacionadas con el desempeño de los graduados en ninguno de los niveles de formación.

$\mathrm{Al}$ igual que cuando se analiza el desempeño según genero, tener ingresos familiares superiores a cinco salarios mínimos resultó estar positivamente asociado con el desempeño. Otra variable que resultó común, pero solo para el nivel técnico y tecnológico, es el origen de la IES; para los estudiantes de programas técnicos y tecnológicos, estudiar una institución privada está asociado negativamente con su desempeño académico en la educación superior. Igualmente, la variable tipo de institución, particularmente escuela/institución tecnológica vs universidad, está asociada con el desempeño de los estudiantes de programas tecnológicos y profesionales. Estudiar programas tecnológicos y profesionales en una escuela/institución tecnológica, en vez de una universidad, está asociado negativamente con el desempeño académico del estudiante.

Con respecto a las demás variables utilizadas, los resultados son diferentes según el nivel de formación analizado.

\section{Conclusiones}

Es importante recalcar que los resultados aquí presentados solo hacen referencia a la muestra objeto de este estudio y que, debido a las características de esta, no hace generalizaciones para la población colombiana.

Para concluir, se puede destacar que, sin importar el nivel de formación o el género de los estudiantes, el desempeño académico previo, medido en el resultado de las prueba Saber 11 en las áreas de química, física, matemáticas, lenguaje, filosofía, biología e idiomas, está fuertemente asociado con el desempeño académico de los estudiantes que presentaron la prueba Saber 2009. Este resultado confirma resultados de otras investigaciones, nacionales e internacionales, en las que se analiza el tema: Ajiboye y Tella (2007), Cheesman, Simpson y Wint (2006), entre otros.

De hecho, en este estudio, el desempeño académico previo explica aproximadamente la mitad de la varianza del desempeño en la educación superior y tiene su mayor poder explicativo para 
los estudiantes que estudian programas profesionales y el menor para las mujeres. Mientras tanto, las variables socio-económicas y educativas tienen un poder explicativo menor que el desempeño académico previo de los estudiantes.

Cuando se desagregan los resultados de las características de la educación, se encuentra que de este grupo de variables, aquellas relacionadas con las características de la educación secundaría, entiéndase, categoría del colegio, naturaleza del colegio y tipo de diploma de bachillerato, aportan poco a la explicación del desempeño en la educación superior.

Está situación indicaría que, más allá de las características académicas de la educación secundaria, estudiantes con buen desempeño académico durante el bachillerato, probablemente, seguirán teniendo un buen desempeño durante sus estudios superiores.

Otro aspecto por resaltar de las variables educativas es el relacionado con el tipo de institución en la cual los estudiantes cursan sus estudios. En general, en los análisis hechos, ya sea por género o nivel de formación, se encontró una asociación moderada y negativa entre tipo de IES y desempeño académico; estudiar en una IES diferente a la universidad está asociado negativamente con el desempeño de los estudiantes que cursan programas tecnológicos y profesionales. De manera similar, estudiar en un insti- tuto o escuela tecnológica está asociado negativamente con el desempeño de los estudiantes que cursan programas técnicos profesionales.

El hecho de que el tipo de IES esté relacionado con el tipo de programa estudiado muestra la importancia de la existencia de los diferentes tipos de instituciones, pues ellas están asociadas con el desempeño de sus estudiantes de manera diferente. Además, la existencia de IES especializadas en la oferta de diferentes niveles de formación serviría para ser más eficiente y ser capaces de responder de una forma más acertada las necesidades del mercado.

Igualmente, este resultado demuestra la importancia que tienen las ITP frente a las universidades en la formación técnica profesional, por lo que se hace necesario desarrollar acciones para incentivar el mejoramiento de la calidad, pues ello ayudaría a consolidarlas a estas y a sus programas en la sociedad colombiana.

Finalmente, vale la pena resaltar que, a pesar de que en los últimos años se han incrementado las investigaciones sobre el tema de la educación superior, el análisis de la educación superior por género y nivel de formación, entiéndase, técnica profesional, tecnológica y profesional, es todavía escasa.

Este trabajo intenta contribuir al análisis de esta temática; sin embargo, son necesarias más investigaciones que aborden el tema. En 
especial, este estudio muestra ciertas áreas en las que valdría la pena profundizar, no solo porque la información es escasa, sino por su relevancia para apoyar el crecimiento del sistema de educación superior. Uno de ellos es analizar el desempeño en la educación superior de los graduados de la educación secundaria teniendo como referencia la categoría Icfes de la institución; en particular, la de bachilleres graduados de colegios clasificados en las categorías media y baja, esta información daría luces para generar estrategias para preparar un capital humano que podría estarse desperdiciando, ya sea por dificultades de ingreso a la educación superior o por su baja permanencia en el sistema.

Igualmente, el poco aporte explicativo de las variables socioeconómicas y educativas aquí utilizadas deja pensar que existen otras variables, por ejemplo, variables relacionadas con las características psicológicas de los estudiantes, con los métodos de enseñanza y los métodos de aprendizaje utilizados en la educación superior, que podrían ayudar a explicar el desempeño académico de los estudiantes de la educación superior.

\section{Referencias bibliográficas}

Ajiboye, J. y Tella, A. (2006). Class Attendance and Gender Effects on Undergraduate Students' Achievement in a Social Studies Course in Botswana. Essays in Education, 18.

Armenta, N., Pacheco, C. y Pineda, E. (2008). Factores socioeconómicos que intervienen en el desempeño académico de los estudiantes universitarios de la Facultad de Ciencias Humanas de la Universidad Autónoma de Baja California. Revista de Investigación en Psicología, 11(1), 153-165.

Blackman, S. (2011). Study Examines Harvard's Academic Gender Gap. Recuperado de http://www.usnews.com/education/blogs/MBA-admissions-strictly-business/2011/05/06/ study-examines-harvards-academic-gender-gap

Cheesman, J., Simpson, N. y Wint, A. (2006). Determinants of Student Performance at University: Reflections from the Caribbean. Jamaica.

Conger, D. y Long, M. C. (2010). Why are Men Falling Behind? Gender Gaps in College Performance and Persistence. The Annals of the American Academy of Political and Social Science, 627(1), 184-214. 
Duarte, M. y Jesús, G. (2006). Predictores del Desempeño académico en el primer año de universidad en una institución pública estatal. Puebla, Mexico.

Felder, R., Felder, G., Mauney, M., Hamrin, C. y Dietz, J. (1995). A longitudinal Study of Engineering Student Performance and Retention. Journal of Engineering Education, 84(2), 151-163.

Ferreyra, M. (2007). Determinantes del desempeño universitario: efectos heterogéneos en un modelo censurado. (Tesis de Maestría en Economía). La Plata, Argentina.

Figueroa, M. (2000). Making Sense of Male Experience The Case of Academic Underachievement in the English-speaking Caribbean. IDS Bulletin, 31(2), 68-74.

Garbanzo, G. (2007). Factores asociados al rendimiento académico en estudiantes universitarios, una reflexión desde la calidad de la educación superior pública. Revista Educación, 31(1), 43-63.

Garzón, R., Rojas, M., Del Riesgo, L. y Salamanca, A. (2010). Factores que pueden influir en el renidimiento académico de estudiantes de Bioquímica que ingresan en el programa de Medicina de la Universidad del Rosario-Colombia. Educación Médica, 13(2), 85-96.

Gomez, V. (2003). Educación Técnica y Tecnológica. Revista Javeriana (694), 28-35.
Hernández, J., Márquez, A. y Palomar, J. (2006). Factores asociados con el desempeño académico en el EXANI-I. Revista Mexicana de Investigación Educativa, 11(29), 547-581.

Icfes. (2014a). Icfes. Recuperado d http://www.icfes.gov.co/examenes/saber-pro/informaciongeneral

Icfes. (2014b). Icfes. Recuperado de http://www.icfes.gov.co/informacion-institucional/informacion-general

Icfes. (2014c). Icfes. Recuperado de http://www.icfes.gov.co/examenes/saber-11 o/primer-semestre-2014/a-quienes-se-evalua

Martínez, P., Asmar, M., Silva, L. y Toro, D. (2005). La educación superior universitaria en Colombia. Una deuda por saldar. lesalc-Unesco.

Norusis, M. (2006). SPSS 15.0 Stadistical Procedure Companion. Upper Saddle River: Prentice Hall Inc.

Nowel, A. y Hedges, L. (1998). Trend in gender difference in academic achievement from 1960-1994: an analysis of differences in mean, variance, and extreme sources. Sex Roles, 39 (112), 21-43.

Peralta Díaz, S., Ramírez Giraldo, A. y Castaño Buitrago, H. (2006). Factores resilientes asociados al rendimiento académico en estudiantes pertenecientes a la Universidad de Sucre (Colombia). Psicología desde el Caribe (17), 196-219. 
Ramírez, C. (2008). Technical/vocational higher education: the case of Colombia and Mexico. (Master Thesis). Universidad de Kassel, Kassel.

Schiefelbein, E. y Simmons, J. (1981). The Determinants of School Achievement: A Review of the Research for Developing Countries. Otawa-Canadá: International Development Research Centre.

Simmons, J. y Alexander, L. (1978). The Determinants of School Achievement in Developing Countries: A Review of the Research. Economic Development and Cultural Change, 26(2), 341-357.

Sistema Nacional de la Información de la Educación Superior (SNIES). (s. f.). SNIES. Recuperado de http://www.mineducacion.gov.co/sistemasdeinformacion/1735/w3-article-212400.html

Türüt-Asık, S. y Meltem, D. (2007). Gender Differences in Academic Performance in a Large Public University in Turkey. Higher Education: The International Journal of Higher Education and Educational Planning, 53(2), 255-277.

Vélez Van Meerbeke, A. y Roa González, C. (2005). Factores asociados al rendimiento académico en estudiantes de medicina. Educación Médica, 8(2), 74-82.

Villalba, A. y Salcedo Barragan, A. (2008). El rendimiento académico en el nivel de educación media como factor asociado al rendimiento académico en la universidad. (Tesis Maestría en Educación). Santa Marta, Colombia. 